\title{
Ileal Pouchitis With Endoscopic Pictures
}

\author{
Hassam Ali ${ }^{1}$, Abeera Sarfraz ${ }^{2},{\text { Hadeera } \mathrm{Ali}^{3}}^{3}$ \\ 1. Internal Medicine, East Carolina University, Vidant Medical Center, Greenville, USA 2. Internal Medicine, Federal \\ Medical and Dental College, Islamabad, PAK 3. Internal Medicine, CMH Institute of Medical Sciences, Bahawalpur, PAK
}

Corresponding author: Hassam Ali, alih20@ecu.edu

\begin{abstract}
Ulcerative colitis (UC) is an inflammatory disorder, and almost one-third of UC patients ultimately undergo surgical interventions because of complications or refractory disease. Current restorative proctocolectomy with ileal pouch-anal anastomosis (IPAA) is the standard intervention for severe chronic UC with refractory disease. Several complications associated with this procedure can occur, including anastomotic leak, sepsis, and pouch ischemia. The most frequent long-term complication is pouchitis, an idiopathic inflammatory condition involving the ileal pouch. Presentations may vary but include stool frequency, urgency, incontinence, fatigue, malaise, and fever, less commonly bloody stools. We report a case of ileal pouchitis in a young patient, two years after proctocolectomy with IPAA responsive to antibiotic treatment. Our case supports that imaging studies like flexible sigmoidoscopy are necessary to rule out other disorders in patients with pouchitis.
\end{abstract}

Categories: Internal Medicine, Medical Education, Gastroenterology Keywords: pouchitis, ileal pouchitis, ulcerative colitis

\section{Introduction}

Pouchitis is an inflammatory disorder and a common complication of ileal pouch-anal anastomosis (IPAA), in patients with ulcerative colitis (UC) and familial adenomatous polyposis (FAP) $[1,2]$. The usual presentation includes diarrhea (increased stool frequency) and fecal urgency. Despite an excellent functional outcome after proctocolectomy with IPAA in patients with UC, pouchitis may present in $80 \%$ of patients, with or without extraintestinal manifestations, in a lifetime [3,4]. Pouchitis is more prevalent in patients with FAP than UC, although the age at its incidence remains earlier in UC patients [5]. Due to its occurrence in inflammatory diseases, a genetic component is a probable factor in its incidence. Genetic polymorphisms, especially those of interleukin (IL)-1 receptor antagonist, have been linked to increased risk of pouchitis [6].

Review began 04/14/2021 Review ended 04/26/2021 Published 04/30/2021

\section{(c) Copyright 2021}

Ali et al. This is an open access article distributed under the terms of the Creative Commons Attribution License CC-BY 4.0., which permits unrestricted use, distribution, and reproduction in any medium, provided the original author and source are credited.

\section{Case Presentation}

A 25-year-old female presented with a one-week history of abdominal pain and watery diarrhea. She had a past medical history of papillary thyroid carcinoma with complete thyroidectomy, UC status post colectomy with IPAA (J-pouch). Her pain was most intense in the left lower abdominal quadrant and only relieved by heating pads. She stated an increased frequency of abdominal bowel movements (her regular bowel movements were four/day). She denied any environmental exposures, dietary changes, fevers, chills but stated malaise. The patient was initially placed nil per oral and started on intravenous fluids for dehydration. Her laboratory works up for inflammatory markers, and routine workup was negative, including thyroid panel. Her stool osmolality was $309 \mathrm{mOsm} / \mathrm{kg}$, consistent with secretory diarrhea and abdominopelvic CT scan revealed J-pouch thickening without evidence of bowel obstruction, ascites, adenopathy, or pneumoperitoneum. The Clostridium difficile toxin was negative and stool culture failed to grow anything. Due to her history of UC and surgical intervention, she underwent flexible sigmoidoscopy with biopsy by gastroenterology and was noted to have a patchy area of mild erythematous mucosa (Figure 1). A tentative diagnosis of ileal pouchitis was made due to erythema on sigmoidoscopy.

The patient was started on ciprofloxacin and metronidazole and discharged with a follow-up in the clinic. Her biopsy later revealed severe acute inflammation and degenerative changes without adenomatous dysplasia or malignancy. She received ten days of ciprofloxacin $500 \mathrm{mg}$ twice a day and metronidazole 500 mg twice a day with resolutions of symptoms. 


\section{Cureus}

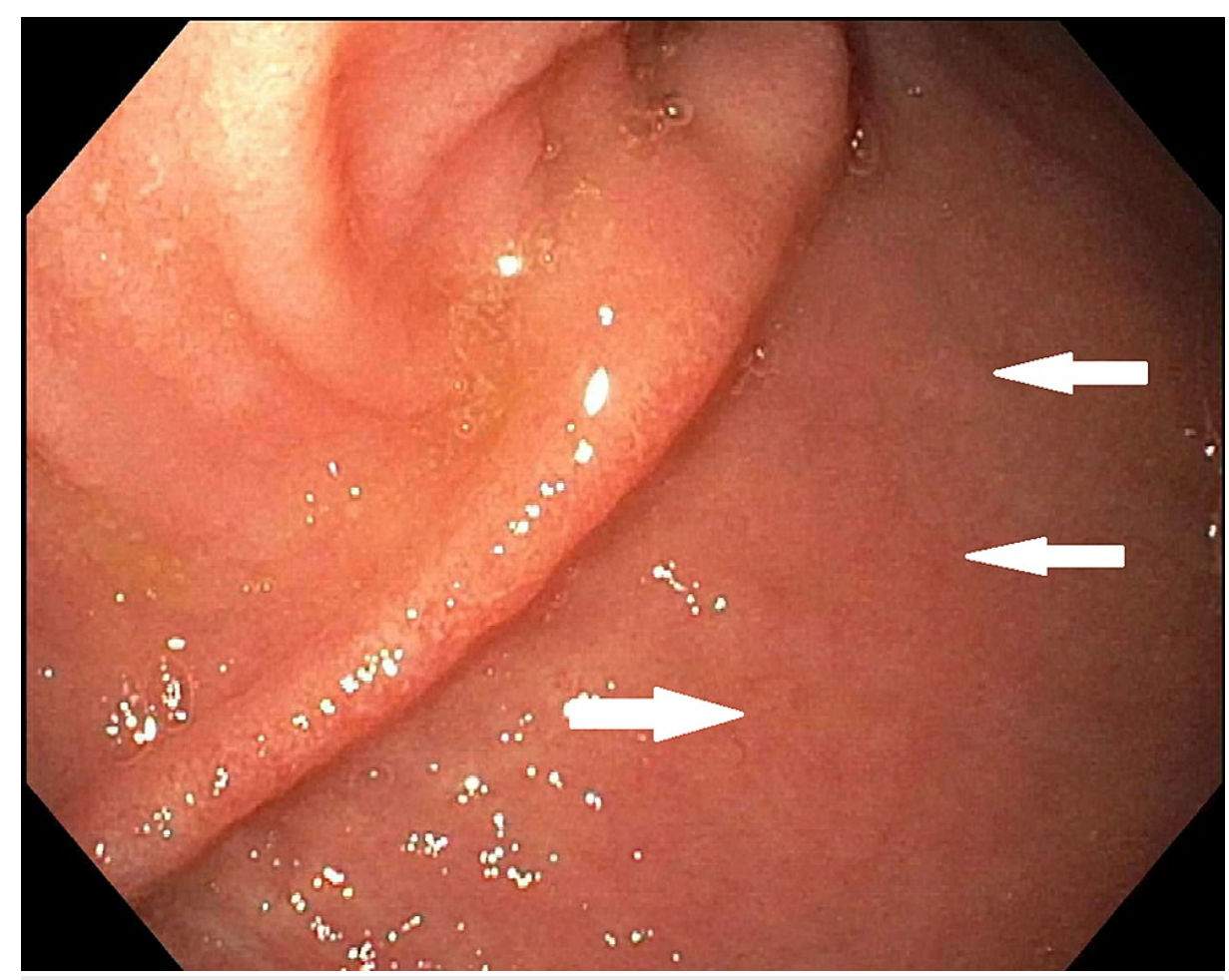

FIGURE 1: Pouchitis after ileal pouch-anal anastomosis ( $\mathrm{J}$-pouch) in ulcerative colitis (white arrows showing erythema).

\section{Discussion}

Pouchitis can present with various clinical manifestations, and patients can have an acute or chronic presentation. The spectrum of presentation ranges from increase stool frequency, urgency, abdominal pain, and fecal incontinence. Patients may have associated pelvic or cramping abdominal pain that may not be relieved by a bowel movement. Despite a lack of bloody bowel movements, pouchitis can result in iron deficiency anemia $[7,8]$. In case of systemic symptoms like fevers, malaise, chills, night sweats, and weight loss, an infectious source of pouchitis should be considered [8].

Ileal pouch endoscopy with biopsy is primarily performed for evaluation in case of a suspected cause of pouchitis. Endoscopically, a clinician may notice erythema (as in our case), erosion or ulceration of mucosa, and increased friability [9]. Typically, additional imaging is not obtained; but to rule out differentials and to assess the presence of mucosal and transmural disease activity, CT scan, pouchography, and MRI can be utilized [10]. Functional abnormalities, for example, pouch manometry, can also be utilized for assessing paradoxical contractions in dyschezia. Pouchitis therapy aims to relieve symptoms and promote mucosal healing. Histologic improvement is emerging as an additional component of disease remission [11]. Treatment response includes improved bowel movement frequency to baseline and better symptom control. Stool culture and susceptibility testing should be done if the patient is not responsive to antibiotics [12]. Typically 14 days of metronidazole $500 \mathrm{mg}$ twice a day or ciprofloxacin $500 \mathrm{mg}$ twice a day can be utilized [12]. No symptomatic recovery despite four weeks of antibiotic therapy can be labeled as antibioticrefractory pouchitis. Therefore, four weeks of combined ciprofloxacin and metronidazole can be given in refractory cases based on susceptibility results $[12,13]$.

In the case of refractory pouchitis, it may be essential to repeat biopsy to exclude any concurrent infection. Blood tests to rule out primary sclerosing cholangitis and stool specimen for Clostridium difficile should also be obtained. Refractory pouchitis is the leading cause for pouch failure, ending in pouch excision or permanent ileostomy. A chronically inflamed pouch can increase the risk of cellular dysplasia or cancer $[14,15]$. Due to antibiotic-based therapy, the management of antibiotic-refractory pouchitis remains a challenge. A high dose of probiotics may effectively prevent pouchitis onset, relapses and can help manage chronic pouchitis refractory to antibiotics [16,17]. Anti-inflammatory agents, biologic therapy, and immunomodulators may also be employed [18].

\section{Conclusions}

Pouchitis is the most prevalent long-term unfavorable sequela of IPAA after restorative proctocolectomy. The natural history of pouchitis is poorly understood. Pouchitis has a wide range of clinical presentations, disease course, and the prognosis is often good. The key to appropriately manage pouchitis is an accurate 
diagnosis and its classification. It is important to consider the cost-effectiveness of diagnostic interventions as well. Treatment of pouchitis is mainly antibiotic-based. Maintenance of remission in antibioticdependent pouchitis and management of antibiotic-refractory pouchitis is a challenge. Secondary causes for refractory pouchitis should be excluded.

\section{Additional Information \\ Disclosures}

Human subjects: Consent was obtained or waived by all participants in this study. Conflicts of interest: In compliance with the ICMJE uniform disclosure form, all authors declare the following: Payment/services info: All authors have declared that no financial support was received from any organization for the submitted work. Financial relationships: All authors have declared that they have no financial relationships at present or within the previous three years with any organizations that might have an interest in the submitted work. Other relationships: All authors have declared that there are no other relationships or activities that could appear to have influenced the submitted work.

\section{References}

1. Haboubi N: Small bowel inflammation in ulcerative colitis . Colorectal Dis. 2006, 8:373-374. 10.1111/j.14631318.2006.01043.x

2. Zezos P, Saibil F: Inflammatory pouch disease: the spectrum of pouchitis . World J Gastroenterol. 2015, 21:8739-8752. 10.3748/wjg.v21.i29.8739

3. Barnes EL, Herfarth HH, Sandler RS, et al.: Pouch-related symptoms and quality of life in patients with ileal pouch-anal anastomosis. Inflamm Bowel Dis. 2017, 23:1218-1224. 10.1097/MIB.0000000000001119

4. Ferrante M, Declerck S, De Hertogh G, et al.: Outcome after proctocolectomy with ileal pouch-anal anastomosis for ulcerative colitis. Inflamm Bowel Dis. 2008, 14:20-28. 10.1002/ibd.20278

5. Quinn KP, Lightner AL, Pendegraft RS, Enders FT, Boardman LA, Raffals LE: Pouchitis is a common complication in patients with familial adenomatous polyposis following ileal pouch-anal anastomosis. Clin Gastroenterol Hepatol. 2016, 14:1296-1301.10.1016/j.cgh.2016.04.010

6. Carter MJ, Di Giovine FS, Cox A, et al.: The interleukin 1 receptor antagonist gene allele 2 as a predictor of pouchitis following colectomy and IPAA in ulcerative colitis. Gastroenterology. 2001, 121:805-811. 10.1053/gast.2001.28017

7. Pastrana RJ, Torres EA, Arroyo JM, Rivera CE, Sánchez CJ, Morales L: Iron-deficiency anemia as presentation of pouchitis. J Clin Gastroenterol. 2007, 41:41-44. 10.1097/01.mcg.0000212641.90477.d0

8. Oikonomou IK, Fazio VW, Remzi FH, Lopez R, Lashner BA, Shen B: Risk factors for anemia in patients with ileal pouch-anal anastomosis. Dis Colon Rectum. 2007, 50:69-74. 10.1007/s10350-006-0752-6

9. Cox BJ, Endler NS, Lee PS, Swinson RP: A meta-analysis of treatments for panic disorder with agoraphobia: imipramine, alprazolam, and in vivo exposure. J Behav Ther Exp Psychiatry. 1992, 23:175-182. 10.1016/0005-7916(92)90034-G

10. Nadgir RN, Soto JA, Dendrinos K, Lucey BC, Becker JM, Farraye FA: MRI of complicated pouchitis. Am J Roentgenol. 2006, 187:W386-W391. 10.2214/AJR.05.1019

11. Kayal M, Plietz M, Radcliffe M, et al.: Endoscopic activity in asymptomatic patients with an ileal pouch is associated with an increased risk of pouchitis. Aliment Pharmacol Ther. 2019, 50:1189-1194. 10.1111/apt.15505

12. Shen B, Fazio VW, Remzi FH, et al.: Combined ciprofloxacin and tinidazole therapy in the treatment of chronic refractory pouchitis. Dis Colon Rectum. 2007, 50:498-508. 10.1007/s10350-006-0828-3

13. McLaughlin SD, Clark SK, Shafi S, Petrovska L, Tekkis PP, Ciclitira PJ, Nicholls RJ: Fecal coliform testing to identify effective antibiotic therapies for patients with antibiotic-resistant pouchitis. Clin Gastroenterol Hepatol. 2009, 7:545-548. 10.1016/j.cgh.2009.01.002

14. Das P, Johnson MW, Tekkis PP, Nicholls RJ: Risk of dysplasia and adenocarcinoma following restorative proctocolectomy for ulcerative colitis. Colorectal Dis. 2007, 9:15-27. 10.1111/j.1463-1318.2006.01148.x

15. Thompson-Fawcett MW, Marcus V, Redston M, Cohen Z, McLeod RS: Risk of dysplasia in long-term ileal pouches and pouches with chronic pouchitis. Gastroenterology. 2001, 121:275-281. 10.1053/gast.2001.26442

16. Gionchetti P, Rizzello F, Poggioli G, et al.: Oral budesonide in the treatment of chronic refractory pouchitis . Aliment Pharmacol Ther. 2007, 25:1231-1236. 10.1111/j.1365-2036.2007.03306.x

17. Fang S, Kraft CS, Dhere T, Srinivasan J, Begley B, Weinstein D, Shaffer VO: Successful treatment of chronic Pouchitis utilizing fecal microbiota transplantation (FMT): a case report. Int J Colorectal Dis. 2016, 31:10931094. 10.1007/s00384-015-2428-y

18. Bernstein CN: Antibiotics, probiotics and prebiotics in IBD . Nestle Nutr Inst Workshop Ser. 2014, 79:83-100. $10.1159 / 000360713$ 